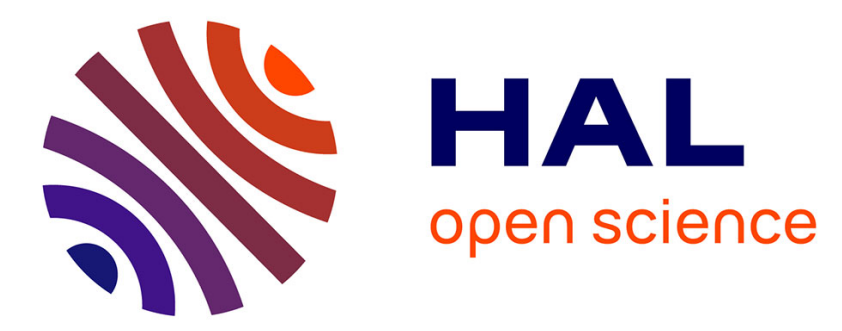

\title{
Utilisation d'un compteur à flux gazeux pour la détection et l'étude d'émissions brèves dans l'ultraviolet extrême \\ P. Dhez, P. Jaegle
}

\section{- To cite this version:}

P. Dhez, P. Jaegle. Utilisation d'un compteur à flux gazeux pour la détection et l'étude d'émissions brèves dans l'ultraviolet extrême. Revue de Physique Appliquée, 1968, 3 (3), pp.275280. 10.1051/rphysap:0196800303027500 . jpa-00242860

\section{HAL Id: jpa-00242860 https://hal.science/jpa-00242860}

Submitted on 1 Jan 1968

HAL is a multi-disciplinary open access archive for the deposit and dissemination of scientific research documents, whether they are published or not. The documents may come from teaching and research institutions in France or abroad, or from public or private research centers.
L'archive ouverte pluridisciplinaire $\mathbf{H A L}$, est destinée au dépôt et à la diffusion de documents scientifiques de niveau recherche, publiés ou non, émanant des établissements d'enseignement et de recherche français ou étrangers, des laboratoires publics ou privés. 


\title{
UTILISATION D'UN GOMPTEUR A FLUX GAZEUX POUR LA DÉTEGTION ET L'ÉTUDE D'ÉMISSIONS BRÈVES DANS L'ULTRAVIOLET EXTREME
}

\author{
Par P. DHEZ et P. JAEGLE, \\ Laboratoire de Chimie Physique de la Faculté des Sciences de Paris, 9r-Orsay.
}

(Reçu le 10 mai 1968, révisé le 9 juillet 1968.)

\begin{abstract}
Résumé. - Parmi les détecteurs de rayonnement utilisables dans l'ultraviolet extrême, les compteurs à flux gazeux de type proportionnel présentent certains avantages. Les auteurs décrivent leur application à l'étude d'émissions brèves en provenance de plasmas produits soit par l'impact d'un faisceau laser sur une cible solide, soit par décharge électrique entre électrodes métalliques. Dans ce dernier cas, la périodicité de l'émission dans le temps est mise en évidence.

Abstract. - Among the radiation detectors which can be used in the extreme U.V. region, the gas-flow proportional type counters have certain advantages. The authors describe their application to the study of short emissions from plasmas, produced, either by impact of a laser beam on a solid target, or by an electrical discharge between metal electrodes. In the latter case the periodicity of the emission as a function of time has been demonstrated.
\end{abstract}

Introduction. - L'amélioration des techniques de détection du rayonnement dans l'ultraviolet extrême et les rayons $\mathrm{X}$ mous $(20 \AA-500 \AA)$ intéresse un domaine étendu de la physique. En effet, la spectrographie des atomes ionisés dont l'émission ultraviolette se produit dans cette région constitue l'un des moyens d'étude des plasmas; d'autre part, l'excitation des couches électroniques peu profondes dans les matériaux solides et dans les gaz donne lieu à l'émission ou à l'absorption de photons dont la détection relève de ces techniques. Enfin, ces mêmes techniques ont un grand intérêt pour les études spatiales dans les rayons $\mathrm{X}$ mous.

Les progrès dépendent notamment de l'adaptation des moyens de détection au régime de fonctionnement de la source qui peut varier considérablement. C'est ainsi qu'un tube à rayons ultra-mous, émettant sous l'effet du bombardement électronique d'une cible, donne un flux constant et relativement faible de photons. Mais le rayonnement intense émis par des électrons accélérés dans un synchrotron est pulsé suivant un cycle dépendant des caractéristiques de la machine et dont la période est mesurée en millisecondes. Dans le cas d'une décharge électrique sous vide, autre moyen classique de produire de l'ultraviolet extrême, la durée de l'émission est très inférieure à une milliseconde; enfin, c'est en dizaines de nanosecondes qu'il faut évaluer la durée d'émission des plasmas produits par l'impact d'un faisceau laser sur une cible solide. A chacune de ces sources correspond une loi particulière de variation d'intensité lumineuse en fonction du temps, et la valeur maximale atteinte par l'intensité varie beaucoup suivant les cas.

La détection par photographie a l'avantage d'être peu sensible à ces différences, mais l'étalonnage d'une émulsion est une opération délicate et la photographie ne permet pas, dans ce domaine, de résoudre facilement dans le temps les émissions intenses et brèves énumérées ci-dessus.

Plusieurs types de détecteurs électroniques peuvent alors être envisagés [1], les photomultiplicateurs étant les plus fréquemment utilisés. Mais l'examen des questions soulevées par de tels détecteurs montre que les compteurs à gaz de type proportionnel présentent un intérêt certain.

Les conditions nécessaires pour une utilisation des compteurs à gaz dans le domaine des rayons $\mathrm{X}$ ultramous sont aujourd'hui bien connues, en particulier grâce aux travaux de A. P. Lukirskii et de ses collaborateurs $[2,3]$. La pression de remplissage doit être basse pour permettre une amplification suffisante des impulsions. Pratiquement, cette pression est de l'ordre de 100 torrs. Elle est donc compatible avec l'emploi de fenêtres très minces et faiblement absorbantes, en collodion par exemple. Placés dans un spectrographe sous vide, des compteurs ainsi réalisés ont un excellent rendement. Il est à noter que la stabilité de leur fonctionnement et leur efficacité ne dépendent pas du tout de la qualité du vide réalisé dans l'installation alors que les photomultiplicateurs sans fenêtre exigent un 
vide propre de l'ordre de $10^{-6}$ torr. La sensibilité des compteurs à gaz varie, en fonction de l'énergie des photons reçus, suivant une loi simple, et le bruit de fond provenant du rayonnement visible parasite est négligeable. De plus, la variété des possibilités dans le choix des mélanges gazeux, de la pression de remplissage et de l'amplification gazeuse donne beaucoup de souplesse aux conditions de leur emploi.

Ges caractéristiques ayant été établies dans le cas de sources à émission continue, nous nous sommes proposés d'étudier le fonctionnement des compteurs à gaz avec des sources à émissions brèves et intenses. Notre but consiste d'une part à déterminer l'intensité émise à une longueur d'onde donnée, par exemple au cours d'une décharge électrique, et d'autre part à résoudre l'émission dans le temps.

Dans les conditions habituelles de leur emploi, les compteurs sont équipés d'une chaîne électronique destinée à trier l'amplitude des impulsions et à effectuer des comptages. On peut ainsi compter jusqu'à $10^{4}$ photons par seconde à $44 \AA \AA$ de longueur d'onde [4] avec un tube à rayons $\mathrm{X}$. Dans le cas où, le compteur étant installé dans un spectrographe, la discrimination d'amplitude n'est pas nécessaire, le nombre de photons compté par seconde peut être plus élevé, mais reste limité par le temps de collection des électrons et des ions. De plus, un compromis entre la constante de temps de l'installation et l'amplification gazeuse est nécessaire : une faible constante de temps favorise un comptage rapide mais demande une grande amplification gazeuse. La charge d'espace devient alors importante au voisinage de l'anode, et le compteur peut cesser d'être proportionnel.

Or le flux incident, lorsque l'on utilise comme source une décharge électrique, peut être des centaines ou des milliers de fois supérieur, pendant un temps très court, à celui délivré par un tube à rayons $\mathrm{X}$ ordinaire dans le même domaine de longueur d'onde. Il est alors impossible, de toute évidence, d'effectuer un comptage d'impulsions. C'est pourquoi nous avons entrepris d'étudier les différentes caractéristiques de la réponse globale du compteur, hauteur et temps de montée par exemple. La simplicité relative des mécanismes d'ionisation dans les gaz permet, il faut le souligner, des prévisions théoriques actuellement impossibles avec les détecteurs solides. Nous avons déjà montré expérimentalement [5] qu'avec un choix convenable du coefficient d'amplification gazeuse, l'impulsion de tension recueillie à la sortie du compteur est proportionnelle à l'énergie totale incidente pendant un cycle complet de l'émission de la source, la constante de temps étant naturellement supérieure à la durée du cycle. Ce travail avait permis de constater que le régime dit de " proportionnalité limitée » apparaît pour des coefficients d'amplification d'autant plus petits que le flux incident est plus grand. D'une façon générale, l'amplification gazeuse doit être nettement plus faible que dans le comptage par impulsions à énergie égale des photons incidents.
En modifiant la constante de temps, nous avons ensuite abordé l'étude de la variation du flux au cours d'un cycle d'émission dans l'ultraviolet extrême d'une étincelle condensée sous vide et déterminé quelques caractéristiques de l'émission du plasma produit par l'impact d'un faisceau laser sur une cible solide. Dans ce dernier cas, la durée de l'émission est de l'ordre de grandeur du temps de collection des électrons dans le compteur, fait qui rend l'interprétation des résultats plus délicate.

Nous décrivons ci-dessous l'appareillage utilisé et les résultats de quelques expériences illustrant les possibilités des compteurs à gaz pour ce type de travaux.

I. Dispositif expérimental. - A. Le spegtrographe. - Le spectrographe que nous utilisons a déjà été décrit par l'un de nous [6]. La figure 1 en donne une

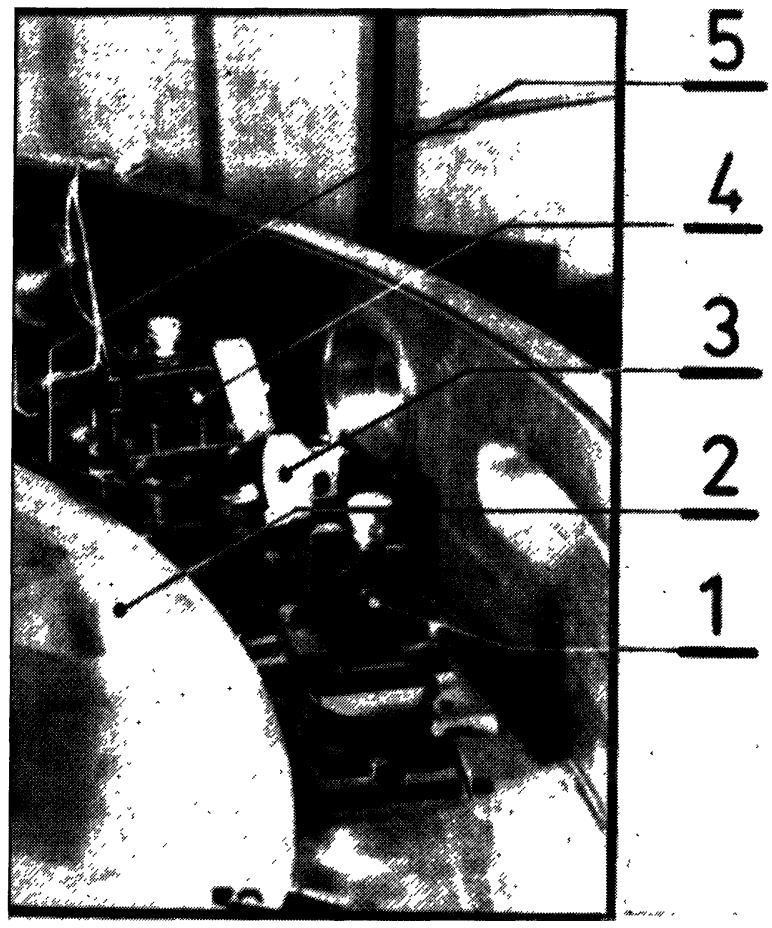

FIG. 1. - Vue d'ensemble du spectrographe et de la plate-forme porte-compteur.

vue partielle. On aperçoit sur cette figure le « banc d'optique circulaire » qui sert de support à tous les éléments du spectrographe. Ces éléments, fente d'entrée, réseaux, détecteurs, peuvent être placés en un point quelconque du banc qui matérialise le cercle de Rowland des réseaux. On dispose donc d'une parfaite souplesse de réglage. En particulier, on peut choisir à volonté l'angle d'attaque du rayonnement sur le réseau, dans un intervalle de $2^{\circ}$ à $40^{\circ}$.

Le montage représenté par la figure 1 comporte une fente source 1 , un réseau 3 et la plate-forme 
porte-compteur 4 utilisée pour le présent travail. Le réseau a un rayon de courbure de $2 \mathrm{~m}$ et possède 576 traits au millimètre. La source de rayonnement a été enlevée pour faciliter la vue des autres éléments.

Les réglages s'effectuent tous en lumière visible grâce à un dispositif optique [6] non visible sur la figure 1. Ce dispositif qui comporte une lunette goniométrique permet d'ajuster l'orientation du réseau avec une précision de $3^{\prime \prime}$ et d'amener le réseau et la fente source sur le cercle de focalisation théorique avec une erreur inférieure à $0,03 \mathrm{~mm}$. Il permet également de mesurer l'angle d'attaque du rayonnement sur le réseau. La fente source est montée sur un support tournant; on peut ainsi la diriger vers la lunette pour les réglages, puis vers le réseau pour le fonctionnement. Sur la figure 1 on aperçoit l'un des orifices de pompage 2 du spectrographe.

B. Plate-forme porte-compteur. - La figure 2 montre la plate-forme porte-compteur vue de dessus. La plate-forme est mobile sur le banc d'optique; elle repose sur le banc par l'intermédiaire de roulements de haute précision. Son entraînement est assuré par un moteur électrique 1 fixé sur le socle, un galet caoutchouté s'appuyant en permanence sur le banc.

A la partie supérieure de la plate-forme sont disposées deux équerres pouvant recevoir chacune un compteur 2. L'une d'elles est escamotable pour permettre la comparaison des réponses de deux détecteurs de types différents. Une fente réglable 3 est placée en avant des compteurs. L'ensemble compteur-fente peut tourner autour de l'axe de la fente. Cette rotation, destinée à orienter le système de détection vers le réseau, est commandée par un moteur électrique 4. Des vis 5 permettent d'amener l'axe de la fente sur l'axe de rotation de la plate-forme. Une vis micro- métrique 6 permet alors de mettre la fente sur le cercle de focalisation. Ces réglages sont effectués à l'aide de la lunette goniométrique comme le réglage de la fente d'entrée du spectrographe.

Pour permettre les repérages sous vide de l'orientation de la position des compteurs, la plate-forme est munie de deux capteurs potentiométriques. Le premier est un capteur circulaire $(5, f i g .1)$. Il est intercalé entre la platine supportant la fente d'entrée des compteurs et le socle roulant, sa définition est de $3^{\prime}$ d'arc. Le second est un capteur linéaire Legpa de $18 \mu$ de définition. Il est fixé au banc par un axe tournant. L'extrémité mobile est solidaire de la plate-forme (7, fig. 2). Ainsi deux mesures de résistance fournissent après étalonnage l'orientation et la position de la fente d'entrée des détecteurs. Pour ces mesures, nous avons formé un pont de Wheatstone avec chaque capteur, et utilisé dans la branche centrale un voltmètre « digital » sensible au millivolt.

L'étalonnage du capteur linéaire, dont dépend la mesure des longueurs d'onde, s'effectue en lumière visible en déterminant pour quelques positions de la plate-forme le long du cercle de focalisation la résistance du capteur et l'angle correspondant mesuré par la lunette goniométrique placée au centre du banc. Il suffit ensuite d'afficher sur l'une des branches du pont de Wheatstone, formé d'une boîte de résistances, la valeur correspondant à la position angulaire désirée et de déplacer la plate-forme à l'aide du moteur électrique jusqu'à annuler la différence de potentiel dans la branche du pont où est disposé le voltmètre. La précision du repérage est de $\pm 0,03 \mathrm{~mm}$ le long du cercle de focalisation. Avec un réseau de 576 traits par $\mathrm{mm}$ et un angle d'attaque de $5^{\circ}$, l'erreur correspondante de longueur d'onde est de $\pm 0,04 \AA$ en premier ordre.

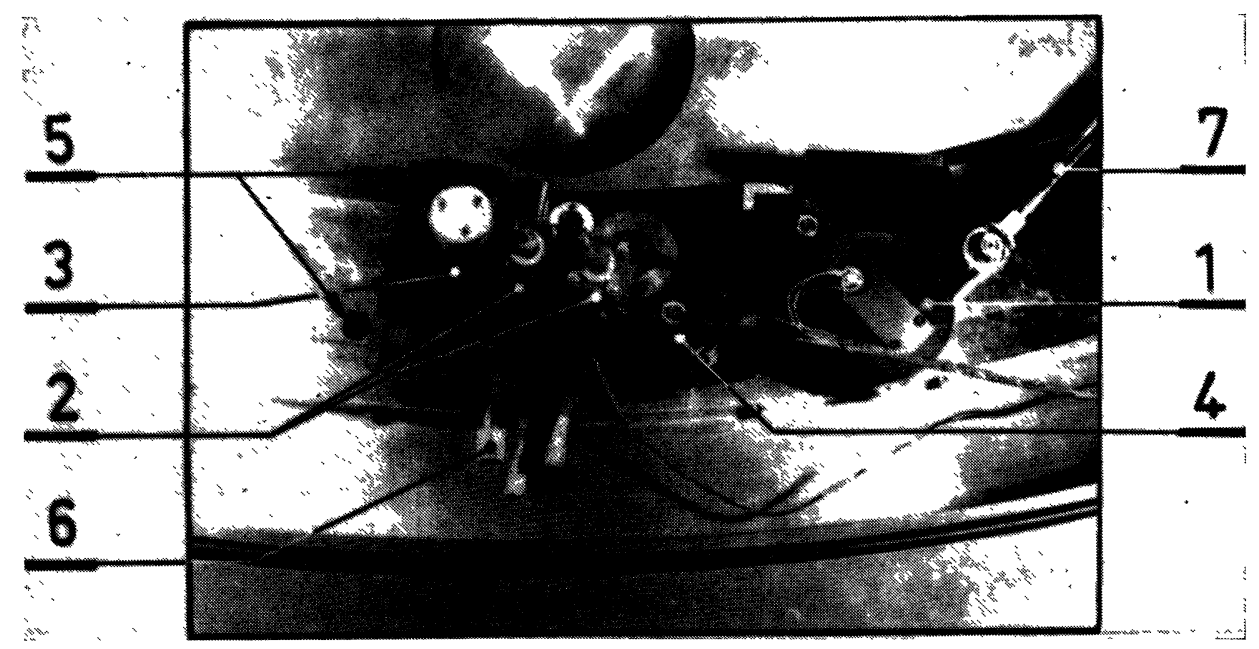

FIG. 2. - La plate-forme porte-compteur vue de dessus. 
C. Le compteur. - Nous utilisons un compteur à flux gazeux de mêmes dimensions que celui décrit dans [2] ( fig. 3). Nous pouvons ainsi comparer plus facilement nos résultats à ceux qui correspondent à
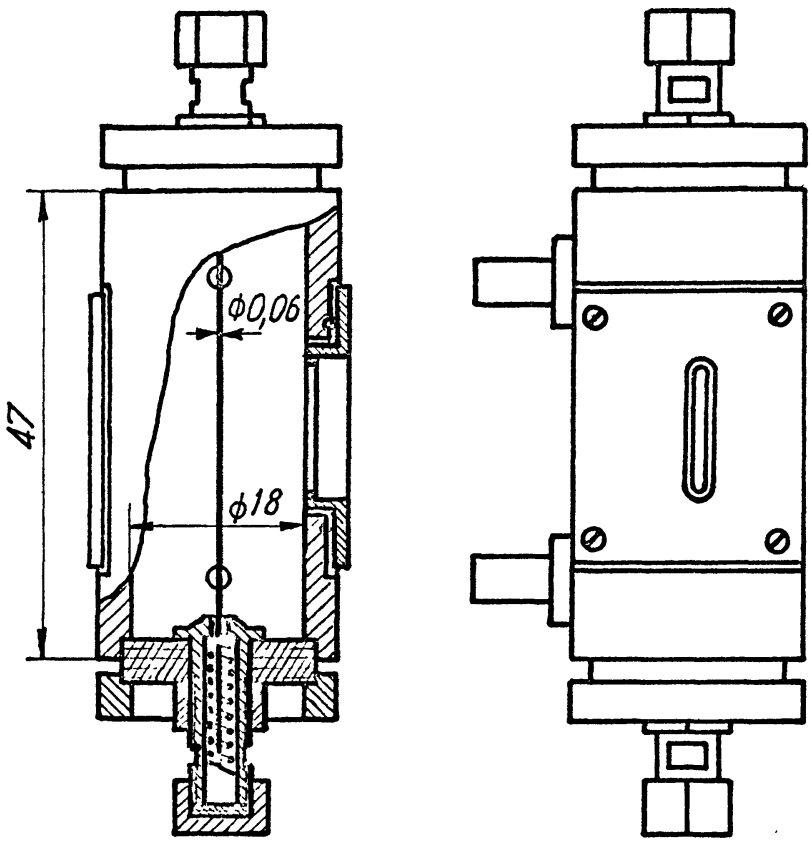

FIG. 3. - Compteur (d'après A. P. Lukirskii, Bull. Acad. Sc. U.S.S.R., Phys. Serie, 1963, 27, 6, 798).

l'emploi d'une source de rayons $\mathrm{X}$ à débit constant comme celle utilisée dans la référence ci-dessus. Le diamètre de la coque est de $18 \mathrm{~mm}$, la hauteur du cylindre est de $47 \mathrm{~mm}$, le filament a $0,06 \mathrm{~mm}$ de diamètre. La fenêtre du compteur est constituée de deux couches superposées de collodion de $1000 \AA$ d'épaisseur. La superposition de ces couches diminue considérablement la porosité des membranes. Pour une hauteur de $8 \mathrm{~mm}$ et une largeur de $0,5 \mathrm{~mm}$, ces fenêtres supportent une pression allant jusqu'à 250 torrs.

La pression habituelle de remplissage avec du méthane est de 64 torrs. Cette pression et la composition du mélange gazeux peuvent être modifiées à volonté grâce à un circuit de verrerie extérieur au spectrographe et relié au compteur par des tubes souples traversant l'enceinte à vide. Un brassage du mélange est assuré en permanence par un agitateur à ailettes. Pour éviter que lors de la mise sous vide du spectrographe une différence de pression importante puisse détruire la fenêtre du compteur, une vanne de communication est insérée entre l'enceinte du spectrographe et le circuit gazeux. Après un pompage mécanique, on effectue un "rinçage " du compteur avec le mélange. Le pompage de l'ensemble est ensuite repris normalement jusqu'à $5 \times 10^{-5}$ torr. Le compteur et le circuit gazeux sont alors isolés du spectrographe et on procède à leur remplissage.

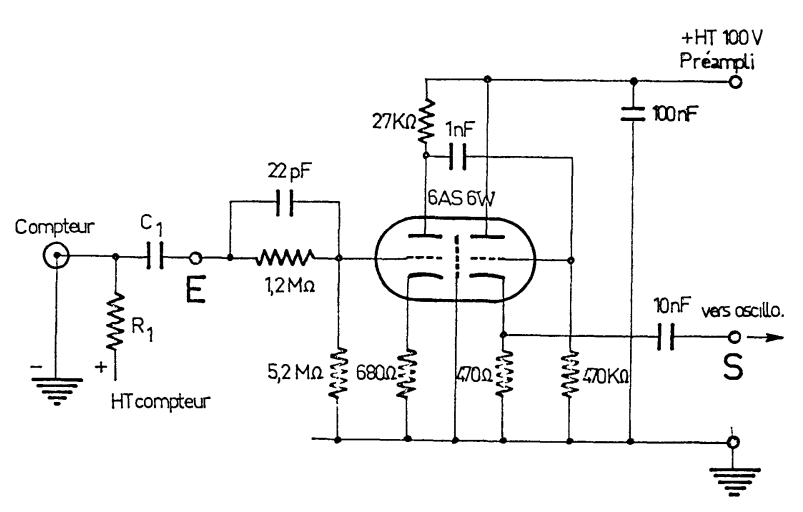

FIG. 4. - Schéma du préamplificateur.

Un préamplificateur très simple à double triode ( fig. 4) est placé près du compteur à l'intérieur du spectrographe. Il permet une adaptation d'impédance avec un gain de 4 environ pour des signaux inférieurs à 2 volts. Le temps de montée du préamplificateur est de l'ordre de 300 nanosecondes et le temps de relaxation du circuit de $10 \mu \mathrm{s}$.

II. Résultats. - Nous avons utilisé deux des sources de rayonnement dont il a été question plus haut pour vérifier le fonctionnement et les possibilités du compteur dans l'ultraviolet extrême.

La figure 5 montre la réponse correspondant à l'émission du spectre continu, à la longueur d'onde

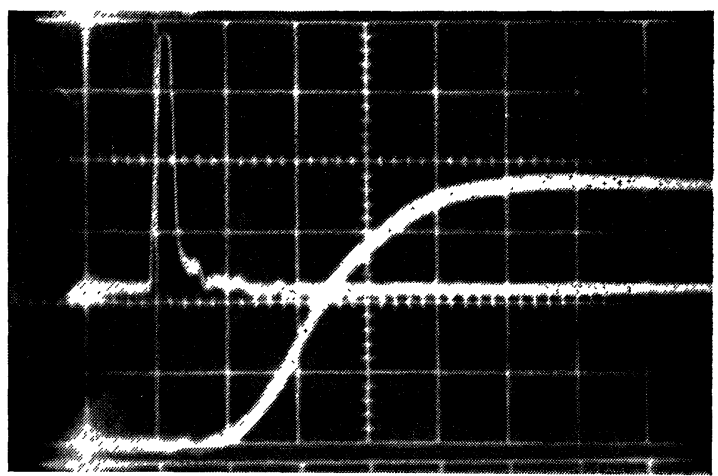

FIG. 5. - Trace supérieure : "pic » laser obtenu au moyen d'une cellule photoélectrique. - Trace inférieure : réponse $\mathrm{du}$ compteur à l'émission, pour $160 \AA$ de longueur d'onde, du plasma produit par le faisceau laser sur une cible métallique.

de $160 \AA$, du plasma produit par l'impact d'un faisceau laser de $45 \mathrm{MW}$ sur une cible d'aluminium. Le laser utilisé a été décrit par ailleurs [7]. La cible est disposée à $50 \mathrm{~mm}$ de la fente d'entrée du spectrographe. Avec une largeur de fente de $20 \mu$ et une ouverture de $15^{\prime}$ pour le faisceau pénétrant dans le spectrographe, un coefficient d'amplification gazeuse de $10^{3}$ suffit pour obtenir un signal de $100 \mathrm{mV}$. 
Le déplacement du compteur permet de tracer des spectres point par point. Afin de tenir compte des variations de la puissance de tir du laser, il est nécessaire de tracer, pour chaque longueur d'onde, la courbe donnant la réponse du compteur en fonction de la puissance de tir. Il résulte des nombreuses déterminations que nous avons effectuées que cette courbe est approximativement linéaire et qu'il suffit généralement de trois tirs pour la tracer avec une précision suffisante. La connaissance de cette droite permet de déterminer la hauteur de l'impulsion correspondant à une puissance choisie. C'est de cette façon qu'ont été déterminés les points portés sur la figure 6 qui montre la courbe obtenue en portant la hauteur de

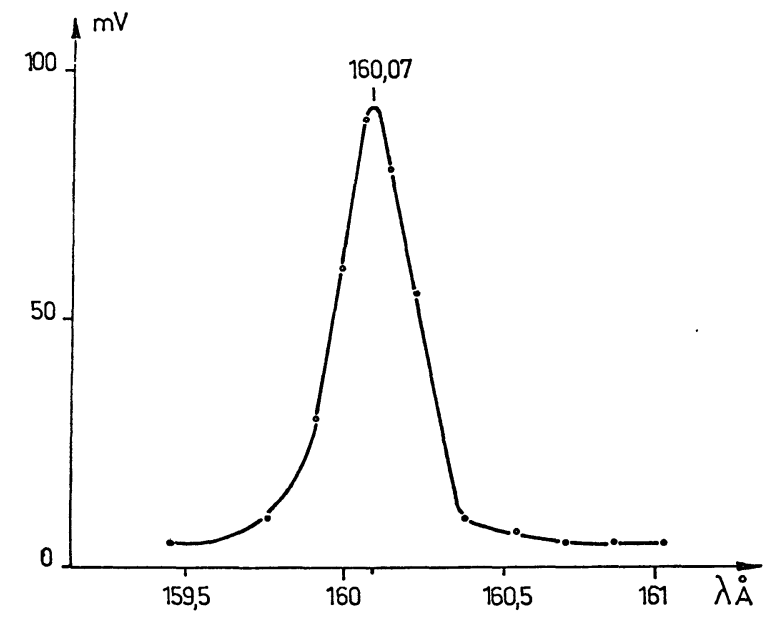

FIG. 6.

Profil de la raie à $160,07 \AA$ du spectre Al-IV.

signal en fonction de la longueur d'onde pour une impulsion laser de $45 \mathrm{MW}$. Elle donne le profil de la raie pour les conditions expérimentales que nous avons définies. Dans ces conditions, la limite de sensibilité du compteur est loin d'être atteinte. En effet, le coefficient d'amplification gazeuse peut être porté à $10^{5}$. Par conséquent, même dans un travail effectué coups par coups, il est possible de diminuer considérablement la largeur des fentes et d'obtenir un pouvoir de résolution élevé.

Une étude àctuellement en cours montre de plus la possibilité d'utiliser ce type de compteur pour mettre en évidence un décalage dans le temps entre l'émission de la raie et celle du continuum de longueur d'onde voisine.

Nous avons obtenu d'autres résultats en utilisant cornme source de rayonnement une étincelle condensée sous vide entre électrodes de niobium et en nous plaçant dans la région de longueur d'onde de $250 \AA$.

La figure 7 montre comment varie la hauteur du signal délivré par le compteur en fonction du flux incident. Pour ces mesures, le préamplificateur décrit n'a pas été utilisé. La liaison entre le compteur et

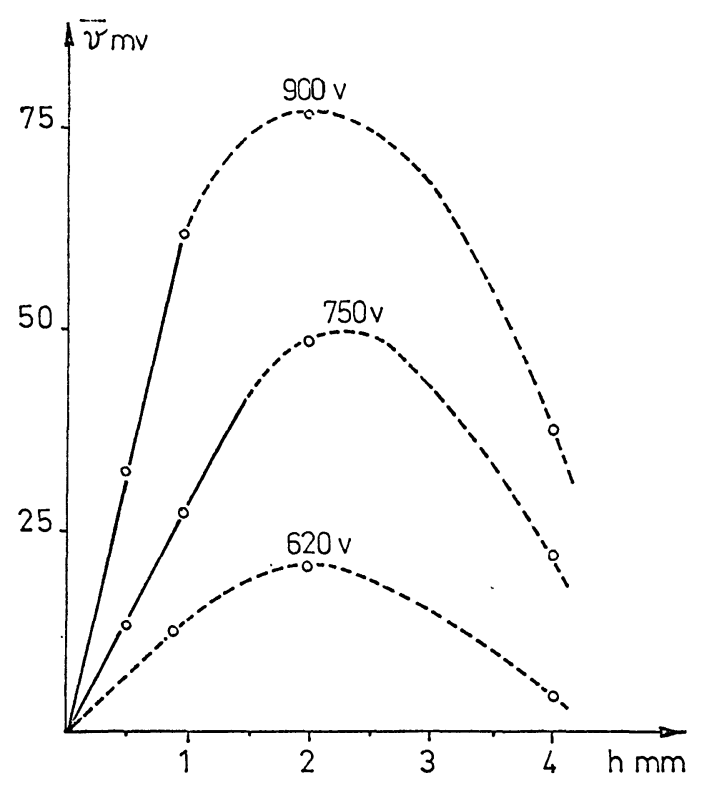

FIG. 7. - Variation de la hauteur du signal compteur en fonction du flux incident.

l'oscillographe était assurée seulement par un câble coaxial de plusieurs mètres; le circuit ainsi constitué formait un intégrateur mais provoquait un affaiblissement du signal de sortie. La variation du flux était obtenue en modifiant la hauteur de la fente d'entrée du compteur (5). Chacune des courbes de la figure 7 présente une partie linéaire définissant les limites dans lesquelles la réponse est proportionnelle au flux. Pour des flux trop importants la variation n'est plus linéaire. En effet, l'augmentation du flux entraîne celle de la densité de charge au voisinage de l'anode centrale du compteur. L'abaissement du champ provoque une chute de l'amplification gazeuse et le défaut de linéa-

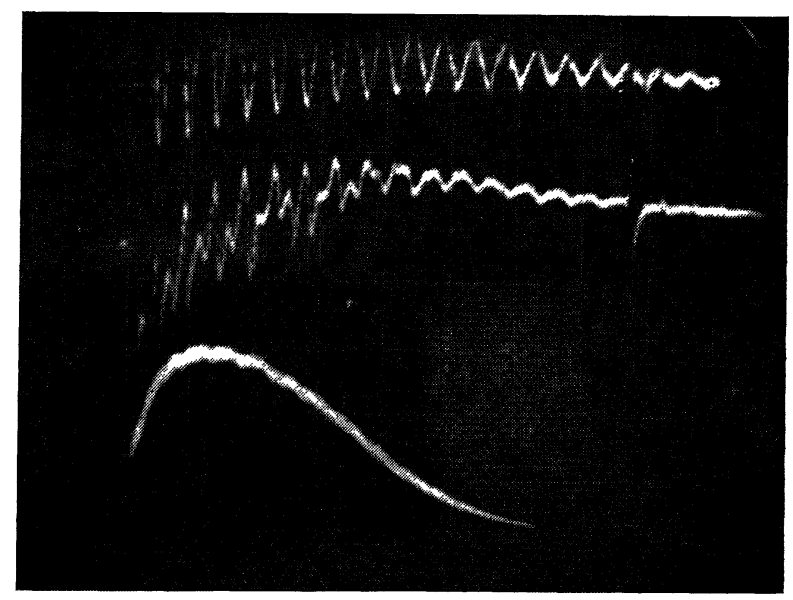

FIG. 8. - Trace supérieure : variation du courant de décharge. - Trace moyenne : variation de l'intensité émise par l'étincelle à $250 \AA$ de longueur d'onde. Trace inférieure : signal intégré. 
rité observé. Il est relativement facile de se prémunir contre ce défaut en s'assurant, par exemple au moyen d'atténuateurs étalonnés, que le compteur travaille dans la partie linéaire des courbes de réponse.

La possibilité de résoudre dans le temps l'émission de la décharge est illustrée par la figure 8. Le circuit de décharge a une capacité de $0,5 \mu \mathrm{F}$ et sa self peut être de 25 ou $50 \mu \mathrm{H}$. La pseudo-période du courant de décharge est respectivement de 20 et $30 \mu$ s environ. La tension d'éclatement de l'étincelle peut varier entre 25 et $80 \mathrm{kV}$. L'installation de détection comporte un intégrateur très simple (fig. 9).

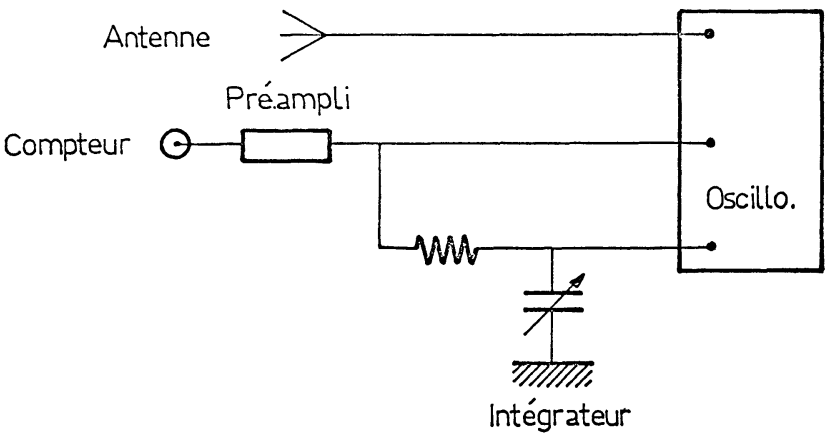

FIG. 9. - Schéma de l'installation de détection.

L'oscillogramme de la figure 8 montre de haut en bas :

- la courbe représentant la dérivée du courant de décharge en fonction du temps, obtenue au moyen d'une antenne placée au voisinage de la self de décharge,

- la variation de l'émission de l'étincelle au cours du temps à $250 \AA \AA$ de longueur d'onde, en utilisant le préamplificateur décrit précédemment dans la figure 4,

- le signal obtenu avec l'intégrateur et dont la hauteur maximale est proportionnelle à la quantité de rayonnement totale reçue par le compteur pendant l'étincelle.

L'étude des oscillogrammes de ce type permet d'affirmer que l'émission de l'étincelle dans l'ultra- violet extrême est périodique, la fréquence étant double de celle du courant de décharge. Cette périodicité n'avait été observée, à notre connaissance, que dans le domaine visible [8].

L'oscillogramme de la figure 10 a été réalisé avec

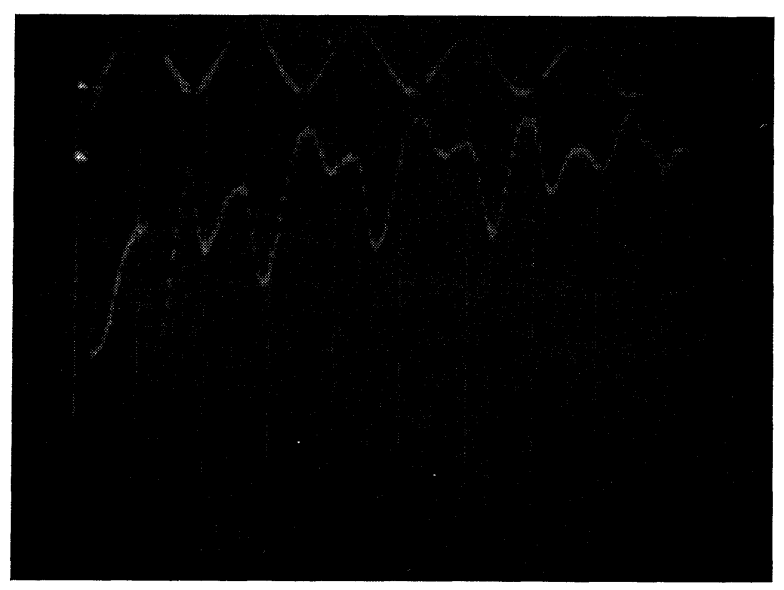

FIG. 10. - Oscillogramme montrant la résolution dans le temps de l'émission de l'étincelle.

une amplification gazeuse élevée pour laquelle la réponse du compteur n'est plus linéaire. C'est pourquoi la décroissance de l'intensité lumineuse après chaque période apparaît comme peu importante. Mais cet oscillogramme montre clairement qu'aux alternances positives et négatives du courant de décharge correspondent des intensités d'émission très inégales suivant la parité de l'alternance. On obtient successivement un maximum élevé puis un maximum faible.

Ces exemples montrent la variété des applications dont les compteurs à gaz sont susceptibles dans le domaine des rayons $\mathrm{X}$ mous et de l'ultraviolet extrême.

Remerciements. - Les auteurs remercient Mlle Y. Cauchois, Professeur à la Faculté des Sciences de Paris, pour l'intérêt constant qu'elle a porté à ce travail et pour l'aide qu'ils ont reçue d'elle en toutes circonstances.

\section{BIBLIOGRAPHIE}

[1] SCHREIDER (E. Ya.), Soviet Physics, Technical Physics, 1965, 9, 12, 1609.

[2] Lukirski (A. P.), Errhow (O. A.) et BryTov (I. A.), Bull. Acad. Sc. U.S.S.R., Physical Series, 1963, 27, 6, 798.

[3] LuKIRSkII (A. P.) et BRYTOV (I. A.), Inst. and Experiment. Techn. U.S.A., 1965, 5, 1083.

[4] LuKirski (A. P.), BryTov (I. A.) et ERShow (O. A.), Bull. Acad. Sc. U.S.S.R., Physical Series, 1963, 27, 3, 447.
[5] Dhez (P.) et Jakgle (P.), C. R. Acad. Sc. Paris, $1^{\text {er }}$ juin 1966, 262, 1432.

[6] JAEGLE (P.), Thèse Doctorat d'État, Paris, 1965. Communication à l'Internationales Symposium Roentgenspektren und chemische Bindung (publié par Wissenschaftlichen Zeitschrift, Leipzig).

[7] BuU Ban, Leach (S.), TaIEB (G.) et Velghe (M.), J. Chimie Physique, 1967, 2, 397.

[8] WAhr (J. C.), MCCORMick (W. W.) et SAWYER (R. A.), J.O.S.A., 1953, 43, 3, 151. 